Gamonales, J.M.; León, K.; Jiménez, A. y Muñoz, J. (2019) Indicadores de rendimiento deportivo en el fútbol-7 para personas con parálisis cerebral / Sport Performance Indicators in Football 7A-Side for People with Cerebral Palsy. Revista Internacional de Medicina y Ciencias de la $\begin{array}{lllllll}\text { Actividad Física y el Deporte vol. } 19 & \text { (74) pp. 309-328 }\end{array}$ Http://cdeporte.rediris.es/revista/revista74/artindicadores1023.htm DOI: http://doi.org/10.15366/rimcafd2019.74.009

\title{
ORIGINAL
}

\section{INDICADORES DE RENDIMIENTO DEPORTIVO EN EL FÚTBOL-7 PARA PERSONAS CON PARÁLISIS CEREBRAL}

\section{SPORT PERFORMANCE INDICATORS IN FOOTBALL 7- A-SIDE FOR PEOPLE WITH CEREBRAL PALSY}

\author{
Gamonales, J.M.. ${ }^{1}$ León, K. ${ }^{1,2}$; Jiménez, A. ${ }^{1}$ y Muñoz-Jiménez, J.1,2 \\ ${ }^{1}$ Facultad de Ciencias de la Actividad Física y el Deporte. Universidad de Extremadura (España) \\ martingamonales@unex.es, fleon@unex.es, ajimenezpaz@gmail.com, suliwan@unex.es \\ 2 Universidad Autónoma de Chile (Chile) jesus.munoz@uautonoma.cl, \\ francisco.leon@uautonoma.cl
}

\section{AGRADECIMIENTOS Y/O FINANCIACIÓN}

Trabajo desarrollado dentro del Grupo de Optimización del Entrenamiento y Rendimiento Deportivo (G.O.E.R.D.) de la Facultad de Ciencias del Deporte de la Universidad de Extremadura.

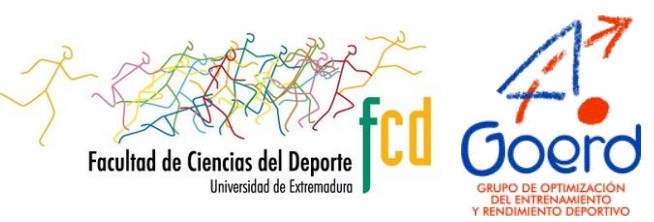

Este trabajo ha sido parcialmente subvencionado por la Ayuda a los Grupos de Investigación (GR15122) de la Junta de Extremadura (Consejería de Empleo e Infraestructuras); con la aportación de la Unión Europea a través de los Fondos Europeos de Desarrollo Regional (FEDER).

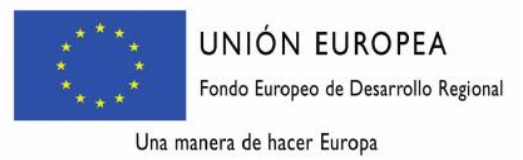

\section{JUNTA DE EXTREMADURA}

Código UNESCO / UNESCO code: 5899 Educación Física y Deportiva / Physical Education and Sports.

Clasificación Consejo de Europa / Council of Europe classification: 17. Otras: Procesos de percepción / Others: Perception processes.

Recibido 23 de junio de 2017 Received June 23, 2017

Aceptado 22 de enero de 2018 Accepted January 22, 2018 


\section{RESUMEN}

El presente estudio pretende determinar los indicadores de rendimiento que influyen en el lanzamiento a portería en fútbol a 7 para personas con parálisis cerebral (Fa7PC). La muestra está compuesta por los lanzamientos de doce partidos seleccionados aleatoriamente de la competición de Fa7PC de los Juegos Paralímpicos de 2012. Los resultados un número elevado de lanzamientos, sobre todo en los últimos minutos del partido, siendo los equipos mejor clasificados los que realizan más. Los jugadores que más lanzan son los delanteros con más capacidad funcional (CP8), a pesar de no ser más efectivos que el resto. El pie derecho y el empeine son las zonas más utilizadas, con trayectorias bajas, oposición cercana y desde zonas centrales del campo.

El lanzamiento con mayor probabilidad de éxito se realiza desde Zona 2, 3 o 6, con el interior del pie, una altura baja y sin oposición defensiva o sólo frente al portero.

PALABRAS CLAVE: fútbol a 7, discapacidad, Juegos Paralímpicos, indicador de rendimiento, análisis de juego.

\section{ABSTRACT}

The aim of the study is determining performance indicators that influence in the results of shot on goal in football 7-a-side for persons with cerebral palsy (Fa7CP). The sample was composed of all shots on goal executed during twelve matches of different competition phases of the Fa7PC Paralympic Games in London 2012. The results show a high number of shots, especially in the last minutes of the match. Best-ranked teams are the most throwers and front players with more functional capacity (CP8) have the biggest offensive weight, despite not being more effective than other players. Right foot and instep are the most used areas to shot on goal, which more frequently have low trajectories, develop with the close opposition and from the field central areas.

The launch with the highest probability of success is executed from Zone 2, 3 or 6 , with the inside of the foot, a low height and without defensive opposition or only in front of the goalkeeper.

KEY WORDS: football 7-a-side, disability, Paralympic Games, performance indicator, match analysis.

\section{INTRODUCCIÓN}

La parálisis cerebral (en adelante, PC) es un trastorno persistente del movimiento y de la postura, causado por una lesión no evolutiva del sistema nervioso central (Boyd \& Graham, 1999). En función de la gravedad y el tipo de PC, puede verse afectada la función o estructura del cuerpo (Kloyiam, Breen, Jakeman, Conway \& Hutzler, 2011). Por tanto, existen distintas formas de PC en función de la afectación motora, siendo la espasticidad (García-Ribés, 2004) y la 
debilidad muscular (Bruyère, VanLooy \& Peterson, 2005) las manifestaciones más frecuentes. En el caso de las personas con PC, una de las modalidades deportivas con mayor interés y práctica entre este colectivo es el fútbol a 7 (en adelante, Fa7PC) muy similar al fútbol convencional (CPISRA, 2014). Es un juego que enfrenta siete jugadores de cada equipo en dos períodos de treinta minutos y con un descanso de diez minutos. No hay fuera de juego a trece metros de la línea de fondo y los saques de banda pueden hacerse con una sola mano (IFCPF, 2016).

Los jugadores, para poder competir, tienen que tener una mínima discapacidad definida por las diferentes organizaciones, que le provoque una desventaja que impida su participación en deporte normalizado (Tweedy \& Valandewijck, 2011). Con el objetivo de minimizar el impacto de la deficiencia que presenta el jugador en la competición, la CPISRA (Cerebral Palsy International Sports and Recreation Association) desarrolló un sistema de clasificación funcional con ocho clases deportivas: cuatro clases (C1-C4) para atletas de silla de ruedas y las otras cuatro (C5-C8) para atletas ambulantes (Reina, 2014). Las últimas cuatro clases albergan los deportistas elegibles para practicar el fútbol.

La presencia de investigaciones en torno al Fa7PC es reciente y escasa en la literatura científica. Roquetti \& Fernandes (2004) comparan la dermatoglifía, el somatotipo y el consumo máximo de oxígeno de los jugadores de la selección brasileña de Fa7PC. Por otro lado, Andrade, Fleury \& Silva (2005) ponen de manifiesto que la flaqueza muscular, la asimetría de fuerzas y el desequilibrio entre los músculos antagonistas son los principales factores de riesgo de lesión, tras analizar a la selección paraolímpica brasileña de fútbol. Yanci et al., (2014) cuantificaron la altura del salto de los jugadores de Fa7PC en la realización del salto sin y con contramovimiento, observando las relaciones de las variables antropométricas y de la clase funcional con el tiempo del vuelo. Sin embargo, no se han encontrado trabajos científicos sobre indicadores de rendimiento deportivo específicamente centrados en Fa7PC.

El análisis de los indicadores de rendimiento es reciente y emergente, que en poco tiempo ha ganado popularidad como concepto entre los investigadores y profesionales del deporte (Drust, 2010). Es un método de registro y análisis del contexto deportivo (Hughes \& Franks, 2004) que permite dar un feedback cuantitativo y cualitativo óptimo, así como proporcionar datos relevantes del deporte analizado (Hughes \& Franks, 2008). El principal objetivo es identificar fortalezas que pueden ser desarrolladas en el entrenamiento, $y$ debilidades que puedan ser mejoradas antes de la competición. Del mismo modo, el análisis del rendimiento del oponente puede ser utilizado para contrarrestar sus fortalezas y explotar sus debilidades (Lago, 2008). Por ello, un indicador de rendimiento es una selección o combinación de variables de movimiento que tiene el objetivo de definir algunos, o todos, los aspectos del rendimiento de un contexto deportivo (Hughes \& Bartlett, 2008).

Estos indicadores constituyen un perfil del rendimiento ideal, que debe estar presente en la actividad deportiva para conseguir este rendimiento y pueden ser usados como una manera de pronosticar el futuro de actividades 
deportivas (O'Donoghue, 2010). Este tipo de análisis se ha aplicado en deportes de personas con discapacidad, como es el caso de Molik, Kosmol, MorgulecAdamowicz, Laskin, Jezior \& Patrzatek (2009), donde analizan la eficacia de juego en el baloncesto en silla de ruedas o de Morato, Da Cunha, Gamero, Magalhães \& Almeida (2017), en el que desarrollan y evalúan un sistema de observación para el análisis de partidos en goalball. Además, son frecuentes los estudios sobre indicadores de rendimiento en relación a los deportes para personas sin discapacidad, centrados en el proceso del juego, como el de Ibáñez, Lozano \& Martínez (2001) que analizan el tiro a canasta en baloncesto, Manzano, Pacheco \& Lorenzo (2006) que estudian el pase o Mendes \& Tavares (2004) que además se centra en la defensa. En todos estos trabajos se emplea la metodología observacional para conseguir información precisa.

Con respecto al lanzamiento a canasta, y según Ibáñez, García, Feu, Parejo \& Cañada (2009), en los últimos años ha sido objeto de estudio con el fin de encontrar su relación e importancia en el juego. Algunas de las variables empleadas en trabajos específicos de análisis de rendimiento, han sido la zona del lanzamiento y el rol de los jugadores (Tsitskaris, Theoharopoulos, Galanis y Nikopoulou, 2002) o la presión defensiva y el nivel de los jugadores (Ibáñez et al., 2001). Pero aún así, son pocos los trabajos que analizan la eficacia del lanzamiento exclusivamente (Ibáñez et al., 2007; Ibáñez et al., 2009; Piñero, 2008; Prieto, Pérez \& Gómez, 2013; Tsitskaris et al., 2002).

En otro sentido, Lago, Casáis, Domínguez, Lago \& Rey (2009) confirman que las variables contextuales que más influyen en el redimiendo en el fútbol son: la localización del partido, el marcador y el nivel del oponente. Sin embargo, los deportes de equipo siempre conllevan un cierto margen para lo aleatorio y lo impredecible (Lago, 2005). Aún así, hay estudios que han demostrado que los equipos vencedores en competiciones o torneos son aquellos que tienen un mayor promedio de lanzamientos totales, lanzamientos a portería y eficacia en el lanzamiento (Armatas et al., 2009; Casáis, Lago, Lago, Iglesias \& Gómez, 2011; Szwarc, 2004).

Estos estudios permiten observar las conductas que influyen en el rendimiento deportivo en condiciones de calidad, fiabilidad, validez y precisión (Salas \& Hernández-Mendo, 2016), o realizar análisis sincrónico o concurrente a una fase concreta de estudio (Hileno \& Buscá, 2012). Coincide con uno de los aspectos más relevantes en el análisis del rendimiento deportivo, que no es otro que mostrar la capacidad del estudio para obtener resultados significativos referentes al contexto de la investigación, a través del registro riguroso de las variables definidas previamente.

Por tanto, teniendo en cuenta los antecedentes descritos, el propósito global de la investigación es determinar los posibles factores contextuales que influyen en el rendimiento de los jugadores de fútbol a 7 para personas con discapacidad, tomando como referencia del rendimiento el Resultado del lanzamiento. Para ello, el objetivo general se operativiza en dos objetivos específicos: i) describir la modalidad deportiva del Fa7PC de élite; y, ii) realizar una análisis descriptivo e inferencial para determinar la relación entre las variables independientes y dependientes del estudio. 


\section{MÉTODO}

\section{Diseño}

El diseño de investigación planteado para el presente trabajo fue de tipo cualitativo-descriptivo (Montero \& León, 2007), utilizando metodología observacional, donde los datos se han obtenido empleando análisis notacional.

\section{Muestra}

La muestra estuvo compuesta por todos los lanzamientos a portería $(n=444)$ realizados durante doce partidos seleccionados aleatoriamente de las diferentes fases de la competición de Fa7PC de los Juegos Paralímpicos de Londres 2012 (Tabla I).

Tabla I. Análisis descriptivo de la variable partidos.

\begin{tabular}{clcc}
\hline Partido & Fases & Frecuencia & Porcentaje \\
\hline Argentina - Irán & Fase clasificatoria (Grupo 1) & 39 & 8,8 \\
Argentina - Holanda & Fase clasificatoria (Grupo 1) & 34 & 7,7 \\
Irán - Rusia & Fase clasificatoria (Grupo 1) & 39 & 8,8 \\
Rusia - Holanda & Fase clasificatoria (Grupo 1) & 29 & 6,5 \\
Brasil - Ucrania & Fase clasificatoria (Grupo 2) & 34 & 7,7 \\
Gran Bretaña - Brasil & Fase clasificatoria (Grupo 2) & 25 & 5,6 \\
Gran Bretaña - Ucrania & Fase clasificatoria (Grupo 2) & 53 & 11,9 \\
USA - Brasil & Fase clasificatoria (Grupo 2) & 46 & 10,4 \\
USA - Gran Bretaña & Fase clasificatoria (Grupo 2) & 35 & 7,9 \\
Brasil - Irán & Tercer y cuarto puesto & 46 & 10,4 \\
Holanda - Argentina & Quinto y sexto puesto & 20 & 4,5 \\
USA - Gran Bretaña & Séptimo y octavo puesto & 44 & 9,9 \\
Total lanzamientos & & 444 & 100,0 \\
\hline
\end{tabular}

\section{Variables}

Para la elaboración del sistema de categorías objeto de análisis (Anguera, 1991), se empleó el procedimiento propuesto por Anguera \& Mendo (2013), basado en entrevistas con expertos, entrenadores españoles de Fa7PC de nivel nacional e internacional, con la finalidad de definir qué acciones se consideraban determinantes (Tabla II). 
Tabla II. Relación de variables y categorías del estudio de PC.

\begin{tabular}{|c|c|c|c|c|c|c|c|c|}
\hline Variables & & & & Categorí & & & & \\
\hline $\begin{array}{l}\text { Clase } \\
\text { funcional }\end{array}$ & CP5 & $\overline{C P 6}$ & CP7 & CP8 & & & & \\
\hline$n$ & 15 & 9 & 243 & 177 & & & & \\
\hline$\%$ & 3,4 & 2,0 & 54,7 & 39,9 & & & & \\
\hline $\begin{array}{l}\text { Rol } \\
\text { jugador }\end{array}$ & Delantero & Medio & Defensa & Portero & & & & \\
\hline$n$ & 130 & 199 & 115 & 0 & & & & \\
\hline$\%$ & 29,3 & 44,8 & 25,9 & 0,0 & & & & \\
\hline $\begin{array}{l}\text { Zona } \\
\text { corporal }\end{array}$ & $\begin{array}{c}\text { Pie } \\
\text { derecho }\end{array}$ & Pie izq. & Cabeza & Otros & & & & \\
\hline$n$ & 251 & 164 & 24 & 5 & & & & \\
\hline$\frac{\%}{\text { Tipo de }}$ & 56,5 & 36,9 & 5,4 & $\begin{array}{r}1,1 \\
\end{array}$ & & & & \\
\hline $\begin{array}{l}\text { Tipo de } \\
\text { golpeo }\end{array}$ & $\begin{array}{c}\text { Pie } \\
\text { interior }\end{array}$ & $\begin{array}{c}\text { Pie } \\
\text { empeine }\end{array}$ & $\begin{array}{c}\text { Pie } \\
\text { puntera }\end{array}$ & $\begin{array}{c}\text { Pie } \\
\text { exterior }\end{array}$ & $\begin{array}{c}\text { Pie } \\
\text { tacón }\end{array}$ & $\begin{array}{l}\text { Cabeza } \\
\text { frontal }\end{array}$ & $\begin{array}{c}\text { Cabeza } \\
\text { lateral }\end{array}$ & Otros \\
\hline$n$ & 104 & 223 & 73 & 12 & 4 & 16 & 2 & 10 \\
\hline$\%$ & 23,4 & 50,2 & 16,4 & 2,7 & 0,9 & 3,6 & 0,5 & 2,3 \\
\hline $\begin{array}{l}\text { Situación } \\
\text { de golpeo }\end{array}$ & $\begin{array}{l}\text { Previo } \\
\text { control }\end{array}$ & $\begin{array}{c}\mathrm{Al} \\
\text { toque }\end{array}$ & & & & & & \\
\hline$n$ & 243 & 201 & & & & & & \\
\hline$\%$ & 54,7 & 45,3 & & & & & & \\
\hline $\begin{array}{l}\text { Altura } \\
\text { Lanz. }\end{array}$ & Bajo & $\begin{array}{l}\text { Media } \\
\text { altura }\end{array}$ & Alto & & & & & \\
\hline$n$ & 209 & 98 & 137 & & & & & \\
\hline$\%$ & 47,1 & 22,1 & 30,9 & & & & & \\
\hline $\begin{array}{l}\text { Oposición } \\
\text { Lanz. }\end{array}$ & $\begin{array}{l}\text { Sin } \\
\text { Opos. }\end{array}$ & Portero & $\begin{array}{l}\text { Opos. } \\
\text { lejana }\end{array}$ & $\begin{array}{l}\text { Opos. } \\
\text { cercana }\end{array}$ & & & & \\
\hline$n$ & 11 & 87 & 143 & 203 & & & & \\
\hline$\%$ & 2,5 & 19,6 & 32,2 & 45,7 & & & & \\
\hline $\begin{array}{l}\text { Situación } \\
\text { de juego }\end{array}$ & $\begin{array}{l}\text { En } \\
\text { juego }\end{array}$ & Penalti & Falta & & & & & \\
\hline$n$ & 408 & 1 & 35 & & & & & \\
\hline$\%$ & 91,9 & 0,2 & 7,9 & & & & & \\
\hline $\begin{array}{l}\text { Resultado } \\
\text { Lanz. }\end{array}$ & Gol & $\begin{array}{c}\mathrm{A} \\
\text { portería }\end{array}$ & Fuera & Otros & & & & \\
\hline$n$ & 63 & 117 & 147 & 117 & & & & \\
\hline$\%$ & 14,2 & 26,4 & 33,1 & 26,4 & & & & \\
\hline
\end{tabular}

Lanz. Lanzamiento; Opos. Oposición; Izq. Izquierdo

Además, se registró la variable Equipo con las categorías: Argentina, Brasil, Gran Bretaña, Irán, Holanda, Rusia, Ucrania y USA; la variable Zona de lanzamiento, definido por un campograma elaborado a tal efecto (Figura I); y la variable Tiempo con los siguientes rangos de apertura: 0 min. a 9 min. 59 seg., 10 min. a 19 min. 59 seg., 20 min. a 30 min., 30 min. a 39 min. 59 seg., 40 min. a 49 min. 59 seg., 50 min. a 60 min., Prórroga y Penaltis. Todas las variables fueron empleadas en el análisis de los indicadores de rendimiento (O'Donoghue, 2010). 


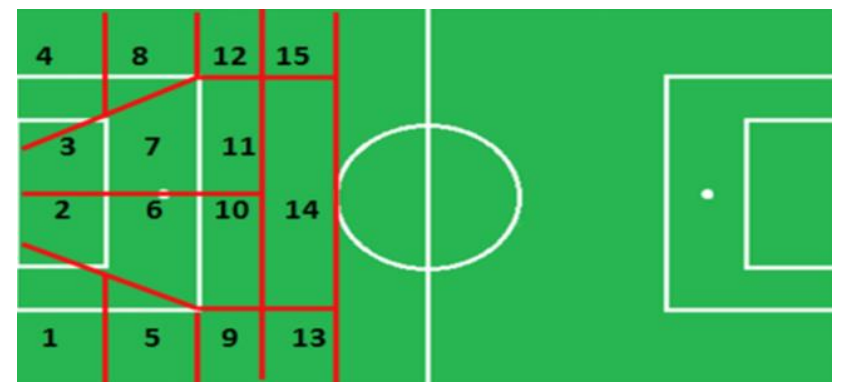

Figura I. Campograma de zona de lanzamiento.

\section{Procedimiento}

La elaboración, diseño y validación de cualquier instrumento de observación en Ciencias de la Actividad Física y del Deporte conlleva seguir un procedimiento metódico y ordenado (Gamonales, León, Muñoz, González, \& Ibáñez, 2018). Por esto, el proceso de investigación se dividió en cuatro fases: Preparatoria; Formación y evaluación de la fiabilidad de las observaciones; Recogida de datos; y por último, análisis estadístico.

Durante la fase preparatoria se desarrolló la planificación, el tratamiento, la organización y la gestión de la información necesaria, que incluía la selección de los medios a emplear en cada una de las etapas de desarrollo del trabajo. También. se incluyó en esta fase el diseño de la formación para la observación de los eventos a registrar (Villarejo, Ortega, Gómez \& Palao, 2014). Tras completar la formación se procedió al cálculo de la fiabilidad intra-observador de los datos recogidos.

\section{Fiabilidad}

Se realizaron dos registros de trece lanzamientos, seleccionados de forma aleatoria entre seis partidos, con una semana de separación entre ambos (Wheeler, Askew \& Sayers, 2010). Para el análisis de la fiabilidad, se utilizó un procedimiento estadístico adaptado del coeficiente Kappa (Cohen, 1960), denominado Multirater Kappa Free (Randolph, 2005), obteniendo un nivel de acuerdo en todas las variables del estudio de $\mathrm{k}>0.88$, que se puede considerar "perfecto" entre medidas repetidas (O’Donoghue, 2010).

Una vez asegurada la fiabilidad de los datos, se procedió a registrar doce partidos seleccionados aleatoriamente entre los veinte que se jugaron en las diferentes fases de la competición de Fa7PC de los Juegos Paralímpicos de Londres 2012, lo que supone un sesenta por ciento de la unidad de análisis.

\section{Análisis estadístico}

Tras la recogida de los datos, se desarrolló un análisis descriptivo de frecuencias y porcentajes. Posteriormente, y debido a que lo datos se han obtenido a partir de variables categóricas, se emplearon modelos matemáticos no paramétricos para el contraste de hipótesis, que permitió estimar la asociación entre las variables, en concreto Chi cuadrado $\left(X^{2}\right)$ y Coeficiente Phi $(\varphi c)$ de Cramer. El nivel de asociación del $\varphi c$ de Cramer se interpretó a través 
de la propuesta de Crewson (2006) y los residuos tipificados corregidos (|1.96|) de las tablas de contingencia (Pardo, 2002).

\section{RESULTADOS}

El análisis descriptivo del estudio muestra que en la mayoría de los partidos de la competición de Fa7PC de los Juegos Paralímpicos de Londres 2012, se producen entre 20-40 lanzamientos. La media de lanzamientos por partido muestra que hay tres equipos que lanzan menos: Argentina $(n=11)$, Holanda $(n=10,66)$, y USA ( $n=10)$; dos equipos que tienen un número similar de lanzamientos: Gran Bretaña $(n=18,5)$, e Irán $(n=18,3)$; y tres equipos que lanzan más a portería que el resto de conjuntos: Ucrania $(n=30,5)$, Rusia $(n=27,5)$, y Brasil $(n=26)$. Con respecto a la variable Clase funcional, destaca que los jugadores que más lanzaron fueron los jugadores clasificados como CP7 ( $n=243)$, seguidos de los CP8 $(n=177)$, mientras que CP5 y CP6 han realizado menos lanzamientos (Gráfico I).

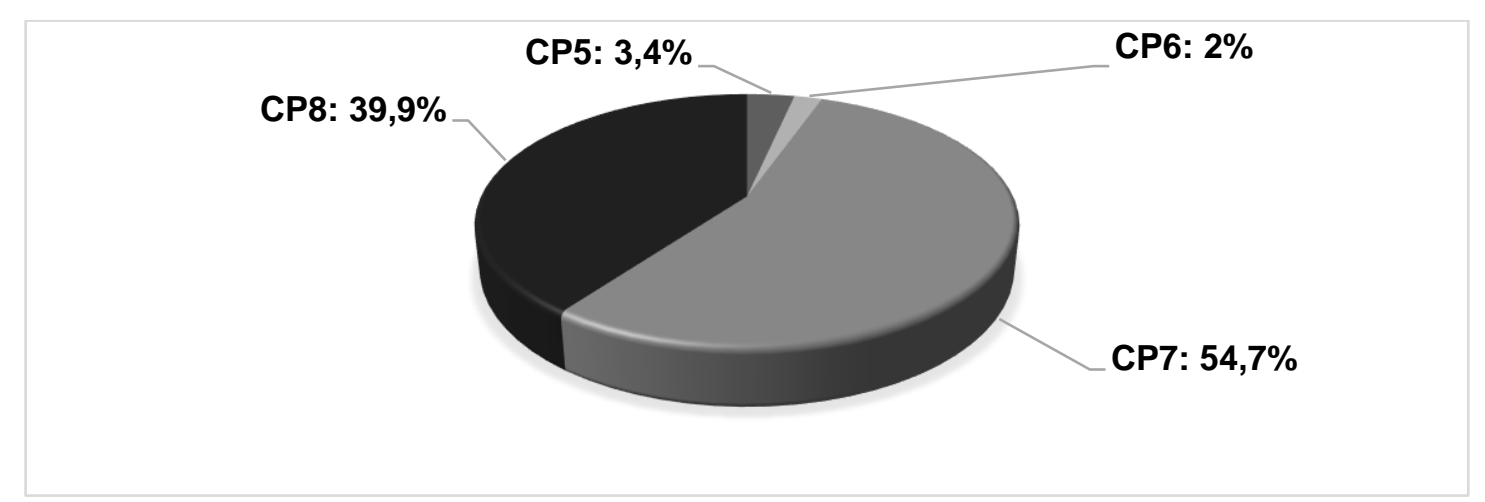

Gráfico I. Porcentajes de lanzamientos por equipo.

En referencia a la variable Tiempo, el momento de juego donde se produjeron menos lanzamientos es en los primeros diez minutos del partido $(n=48)$; mientras que en los últimos diez minutos de partido se ejecutaron el mayor número de lanzamientos $(n=95)$. En el resto de períodos de diez minutos, se realizaron entre sesenta y cuatro y ochenta y tres lanzamientos. En la prórroga sólo se lanzó siete veces a portería. Los jugadores que más lanzamientos realizaron son los centrocampistas ( $n=199)$, seguidos de delanteros $(n=130)$ y, por último, los defensas $(n=115)$. Además, los resultados muestran que la zona del campo en la que más lanzamientos se realizaron fue en la zona $14(n=123)$, seguidos de las zonas $6,7,10$ y 11 . En el resto de zonas se produjeron un número de lanzamientos entre cuatro y trece (Gráfico II). 


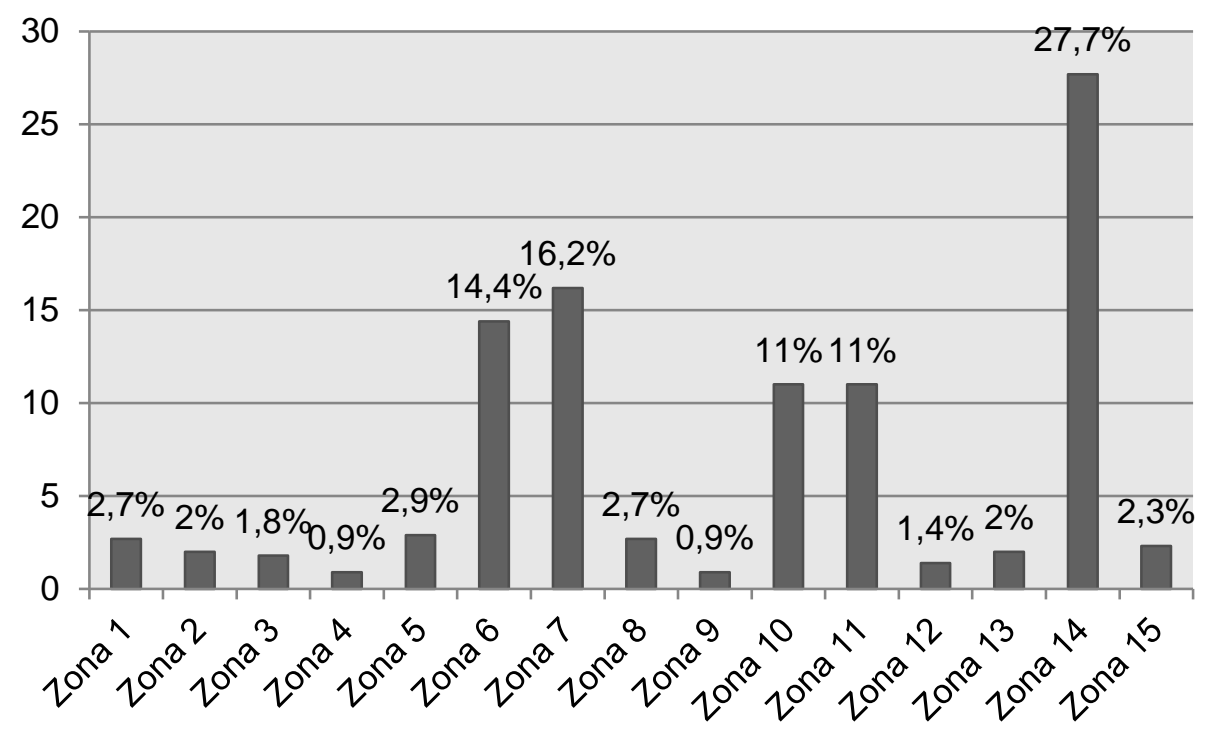

Gráfico II. Porcentaje de lanzamientos por zonas.

Con respecto a la variable Zona corporal, predominaron los lanzamientos con el pie derecho ( $n=251)$, seguido del pie izquierdo $(n=164)$, la cabeza $(n=24)$ $\mathrm{y}$, por último, otro tipo de lanzamientos $(n=5)$. En el gráfico IV se muestran los principales tipos de golpeo de los jugadores con PC.

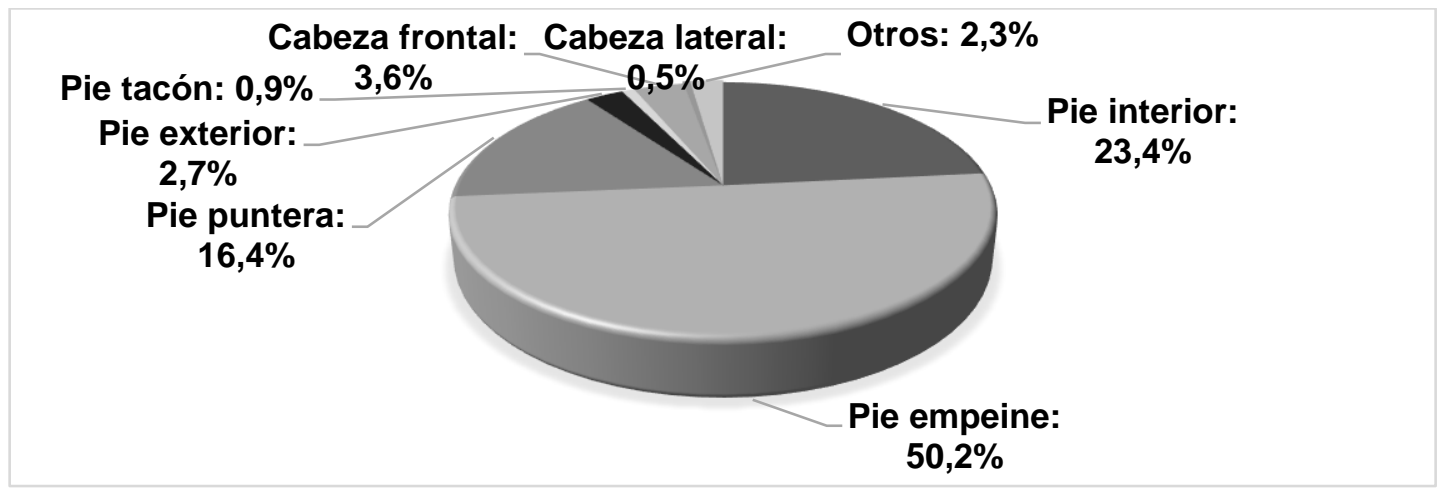

Gráfico III. Porcentajes relacionados con el tipo de golpeo.

La variable Situación de golpeo muestra que los jugadores realizaron un control previo antes de la acción de lanzamiento a portería $(n=243)$. Con respecto a la Altura del lanzamiento, hay un mayor número de lanzamientos bajos $(n=209)$, que altos $(n=137)$, o a media altura $(n=98)$. Estos lanzamientos se produjeron en menor medida sin oposición $(n=11)$, con un jugador solo frente al portero $(n=87)$, o con oposición, lejana $(n=143)$ o cercana $(n=203)$. Atendiendo a la Situación de juego, el mayor número de lanzamientos se produjeron durante el juego $(n=408)$, algunos se realizaron de falta directa $(n=35)$, y sólo se registró un lanzamiento de penalti $(n=1)$.

Por último, en la variable Resultado del lanzamiento se aprecia una gran variabilidad, como se puede observar en el gráfico IV, con un porcentaje de lanzamientos a portería de $26,4 \%$ y un $14,2 \%$ de goles. 


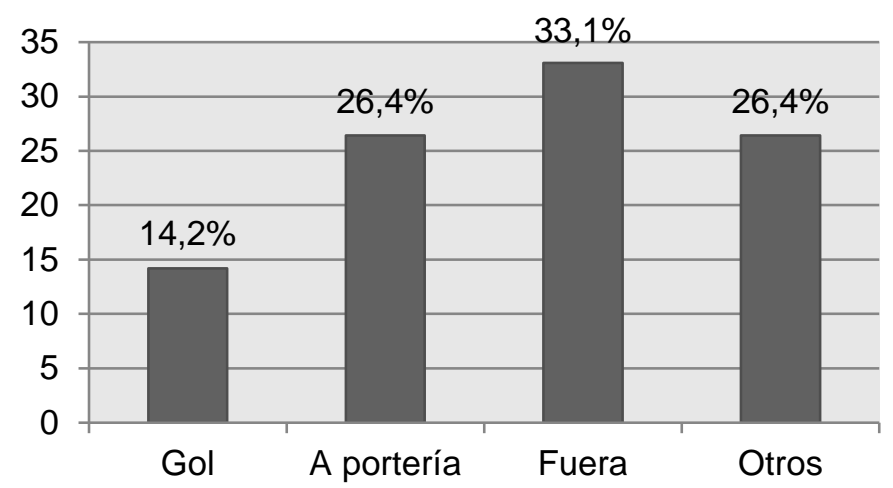

Gráfico IV. Resultado de los lanzamientos a portería.

El resultado del análisis inferencial de la asociación entre variables planteadas en el estudio del Fa7PC, se muestran en la tabla V, en la que se aprecia que hay cinco variables con un grado de asociación significativo: Zona de lanzamiento, Tipo de golpeo, Altura del lanzamiento, Oposición en el lanzamiento y Resultado del lanzamiento con un nivel de asociación moderado $(\varphi c>0,300$ y <0,499); y Situación de juego con un nivel de asociación bajo $(\varphi c>0,100$ y <0,299) (Crewson, 2006). Por otro lado, no existe relación significativa con el resto de variables: Partidos, Equipo, Clasificación funcional, Momento de juego, Rol del jugador, Zona corporal y Situación de golpeo.

Tabla III. Relación entre la variable Resultado de lanzamiento y las variables independientes.

\begin{tabular}{|c|c|c|c|c|c|c|}
\hline \multirow[b]{2}{*}{ Variables relacionadas } & \multicolumn{6}{|c|}{ Juegos Paralímpicos de Londres 2012} \\
\hline & $x^{2}$ & gl. & Sig. & & $\varphi c$ & Sig. \\
\hline Resultado del lanz. / Partidos & 43,403 & 33 & 0,106 & & 0,313 & 0,106 \\
\hline Resultado del lanz. / Equipo & 29,798 & 21 & 0,096 & & 0,259 & 0,096 \\
\hline Resultado del lanz. / Clasificación funcional & 15,270 & 9 & 0,084 & & 0,185 & 0,084 \\
\hline Resultado del lanz. / Momento de juego & 19,301 & 18 & 0,374 & & 0,208 & 0,374 \\
\hline Resultado del lanz. / Rol jugador & 3,734 & 6 & 0,713 & & 0,092 & 0,713 \\
\hline Resultado del lanz. / Zona de lanzamiento & 90,289 & 42 & 0,000 & * & 0,451 & 0,000 \\
\hline Resultado del lanz. / Zona corporal & 9,599 & 9 & 0,384 & & 0,147 & 0,384 \\
\hline Resultado del lanz. / Tipo de golpeo & 43,147 & 21 & 0,003 & * & 0,312 & 0,003 \\
\hline Resultado del lanz. / Situación de golpeo & 7,508 & 3 & 0,057 & & 0,130 & 0,057 \\
\hline Resultado del lanz. / Altura de lanzamiento & 62,181 & 6 & 0,000 & * & 0,374 & 0,000 \\
\hline Resultado del lanz. / Oposición lanzamiento & 82,090 & 9 & 0,000 & * & 0,430 & 0,000 \\
\hline Resultado del lanz. / Situación de juego & 24,662 & 6 & 0,000 & * & 0,236 & 0,000 \\
\hline
\end{tabular}

De una manera pormenorizada, respecto a la relación entre la variable Resultado del lanzamiento y Zona de lanzamiento $\left(x^{2}=90,289 ; p<0,05\right)$, los resultados muestran que existe un nivel de asociación moderado $(\varphi c=0,451)$, y como se puede apreciar en la tabla IV, existe una mayor probabilidad de lo esperado de que los lanzamientos que se producen desde la Zona 1 del campo vayan Fuera de la portería $(R T C=2,5 ; n=8 ; F E=4,0)$ junto con una menor probabilidad de lo esperado de que se produzca Otro resultado $(R T C=-2,1 ; n=0$; $F E=3,2)$. Por otro lado, hay una mayor probabilidad de lo esperado de que los lanzamientos desde la Zona 2 y Zona 3 acaben en $\mathrm{Gol}(R T C=2,6 ; n=4 ; F E=1,3$ y $R T C=2,9 ; n=4 ; F E=1,1)$, al igual que los lanzamientos desde Zona $6(R T C=3,1$; $n=17 ; F E=9,1)$ junto con una probabilidad menor de lo esperado de que los lanzamientos vayan Fuera desde esta zona $(R T C=-2,4 ; n=13 ; F E=21,2)$. Con 
respecto a la Zona 9, también se aprecia una mayor probabilidad de lo esperado de que los lanzamientos obtengan Otro tipo de resultado $(R T C=2,2 ; n=3$; $F E=1,1)$. Finalmente, hay una menor probabilidad de lo esperado de que los lanzamientos desde la Zona 14 del campo acaben en $G o l(R T C=-3,8 ; n=5$; $F E=17,5)$, o Fuera $(R T C=-2,2 ; n=31 ; F E=40,7)$, con mayor probabilidad de lo esperado que se produzca Otro tipo de resultado $(R T C=3,8 ; n=48 ; F E=32,4)$.

Tabla IV. Relación entre las variables Resultado del lanzamiento y la Zona de lanzamiento.

\begin{tabular}{|c|c|c|c|c|c|c|}
\hline \multirow[b]{2}{*}{$\begin{array}{c}\text { Zona de } \\
\text { lanzamiento }\end{array}$} & & \multicolumn{5}{|c|}{ Resultado del lanzamiento } \\
\hline & & Gol & A portería & Fuera & Otros & Total \\
\hline Zona 1 & $\begin{array}{l}\text { n } \\
\text { FE } \\
\text { RTC }\end{array}$ & $\begin{array}{r}2 \\
1,7 \\
0,2\end{array}$ & $\begin{array}{r}2 \\
3,2 \\
-0,8\end{array}$ & $\begin{array}{r}8 \\
4,0 \\
2,5^{*}\end{array}$ & $\begin{array}{r}0 \\
3,2 \\
-2,1^{*}\end{array}$ & $\begin{array}{r}12 \\
12,0\end{array}$ \\
\hline Zona 2 & $\begin{array}{l}\text { n } \\
\text { FE } \\
\text { RTC }\end{array}$ & $\begin{array}{r}4 \\
1,3 \\
2,6^{*}\end{array}$ & $\begin{array}{r}2 \\
2,4 \\
-0.3\end{array}$ & $\begin{array}{r}1 \\
3,0 \\
-14\end{array}$ & $\begin{array}{r}2 \\
2,4 \\
-0.3\end{array}$ & $\begin{array}{r}9 \\
9,0\end{array}$ \\
\hline Zona 3 & $\begin{array}{l}\mathrm{n} \\
\text { FE } \\
\text { RTC }\end{array}$ & $\begin{array}{r}4 \\
1,1 \\
2,9^{*}\end{array}$ & $\begin{array}{r}3 \\
2,1 \\
0,7\end{array}$ & $\begin{array}{r}1 \\
2,6 \\
-1,2\end{array}$ & $\begin{array}{r}0 \\
2,1 \\
-1,7\end{array}$ & $\begin{array}{r}8 \\
8,0\end{array}$ \\
\hline Zona 4 & $\begin{array}{l}\text { n } \\
\text { FE } \\
\text { RTC }\end{array}$ & $\begin{array}{r}1 \\
0,6 \\
0.6\end{array}$ & $\begin{array}{r}2 \\
1,1 \\
1.1\end{array}$ & $\begin{array}{r}1 \\
1,3 \\
-0.3\end{array}$ & $\begin{array}{r}0 \\
1,1 \\
-12\end{array}$ & $\begin{array}{r}4 \\
4,0\end{array}$ \\
\hline Zona 5 & $\begin{array}{l}\text { n } \\
\text { FE } \\
\text { RTC }\end{array}$ & $\begin{array}{r}1 \\
1,8 \\
-0,7\end{array}$ & $\begin{array}{r}6 \\
3,4 \\
1,6\end{array}$ & $\begin{array}{r}5 \\
4,3 \\
0,4\end{array}$ & $\begin{array}{r}1 \\
3,4 \\
-1,5\end{array}$ & $\begin{array}{r}13 \\
13,0\end{array}$ \\
\hline Zona 6 & $\begin{array}{l}\text { n } \\
\text { FE } \\
\text { RTC }\end{array}$ & $\begin{array}{r}17 \\
9,1 \\
3,1^{*}\end{array}$ & $\begin{array}{r}21 \\
16,9 \\
1,3\end{array}$ & $\begin{array}{r}13 \\
21,2 \\
-\mathbf{2 , 4} \mathbf{4}^{*}\end{array}$ & $\begin{array}{r}13 \\
16,9 \\
-1,2\end{array}$ & $\begin{array}{r}64 \\
64,0\end{array}$ \\
\hline Zona 7 & $\begin{array}{l}\text { n } \\
\text { FE } \\
\text { RTC }\end{array}$ & $\begin{array}{r}15 \\
10,2 \\
1,8\end{array}$ & $\begin{array}{r}17 \\
19,0 \\
-0,6\end{array}$ & $\begin{array}{r}27 \\
23,8 \\
0,9\end{array}$ & $\begin{array}{r}13 \\
19,0 \\
-1,7\end{array}$ & $\begin{array}{r}72 \\
72,0\end{array}$ \\
\hline Zona 8 & $\begin{array}{l}\text { n } \\
\text { FE } \\
\text { RTC }\end{array}$ & $\begin{array}{r}0 \\
1,7 \\
-1,4\end{array}$ & $\begin{array}{r}4 \\
3,2 \\
0,6 \\
\end{array}$ & $\begin{array}{r}4 \\
4,0 \\
0,0 \\
\end{array}$ & $\begin{array}{r}4 \\
3,2 \\
0,6 \\
\end{array}$ & $\begin{array}{r}12 \\
12,0\end{array}$ \\
\hline Zona 9 & $\begin{array}{l}\text { n } \\
\text { FE } \\
\text { RTC }\end{array}$ & $\begin{array}{r}0 \\
0,6 \\
-0,8\end{array}$ & $\begin{array}{r}0 \\
1,1 \\
-1,2\end{array}$ & $\begin{array}{r}1 \\
1,3 \\
-0,3\end{array}$ & $\begin{array}{r}3 \\
1,1 \\
2,2^{*}\end{array}$ & $\begin{array}{r}4 \\
4,0\end{array}$ \\
\hline Zona 10 & $\begin{array}{l}\text { n } \\
\text { FE } \\
\text { RTC }\end{array}$ & $\begin{array}{r}6 \\
7,0 \\
-0,4\end{array}$ & $\begin{array}{r}8 \\
12,9 \\
-1,7\end{array}$ & $\begin{array}{r}21 \\
16,2 \\
1,5\end{array}$ & $\begin{array}{r}14 \\
12,9 \\
0,4\end{array}$ & $\begin{array}{r}49 \\
49,0\end{array}$ \\
\hline Zona 11 & $\begin{array}{l}\text { n } \\
\text { FE } \\
\text { RTC }\end{array}$ & $\begin{array}{r}6 \\
7,0 \\
-0,4\end{array}$ & $\begin{array}{r}10 \\
12,9 \\
-1,0\end{array}$ & $\begin{array}{r}20 \\
16,2 \\
1,2\end{array}$ & $\begin{array}{r}13 \\
12,9 \\
0,0\end{array}$ & $\begin{array}{r}49 \\
49,0\end{array}$ \\
\hline Zona 12 & $\begin{array}{l}n \\
\text { FE } \\
\text { RTC }\end{array}$ & $\begin{array}{r}1 \\
0,9 \\
0,2\end{array}$ & $\begin{array}{r}0 \\
1,6 \\
-1,5\end{array}$ & $\begin{array}{r}4 \\
2,0 \\
1,8\end{array}$ & $\begin{array}{r}1 \\
1,6 \\
-0,5\end{array}$ & $\begin{array}{r}6 \\
6,0\end{array}$ \\
\hline Zona 13 & $\begin{array}{l}\mathrm{n} \\
\text { FE } \\
\text { RTC }\end{array}$ & $\begin{array}{r}0 \\
1,3 \\
-1,2\end{array}$ & $\begin{array}{r}1 \\
2,4 \\
-1,0\end{array}$ & $\begin{array}{r}5 \\
3,0 \\
1,4\end{array}$ & $\begin{array}{r}3 \\
2,4 \\
0,5\end{array}$ & $\begin{array}{r}9 \\
9,0\end{array}$ \\
\hline Zona 14 & $\begin{array}{l}\mathrm{n} \\
\text { FE } \\
\text { RTC }\end{array}$ & $\begin{array}{r}5 \\
17,5 \\
-3,8^{*}\end{array}$ & $\begin{array}{r}39 \\
32,4 \\
1,6\end{array}$ & $\begin{array}{r}31 \\
40,7 \\
-\mathbf{2 , 2} \mathbf{2}^{\star}\end{array}$ & $\begin{array}{r}48 \\
32,4 \\
3,8^{\star}\end{array}$ & $\begin{array}{r}123 \\
123,0\end{array}$ \\
\hline Zona 15 & $\begin{array}{l}\mathrm{n} \\
\text { FE } \\
\text { RTC }\end{array}$ & $\begin{array}{r}1 \\
1,4 \\
-0,4\end{array}$ & $\begin{array}{r}2 \\
2,6 \\
-0,5\end{array}$ & $\begin{array}{r}5 \\
3,3 \\
1,1\end{array}$ & $\begin{array}{r}2 \\
2,6 \\
-0,5\end{array}$ & $\begin{array}{r}10 \\
10,0\end{array}$ \\
\hline Total & $\mathrm{n}$ & 63 & 117 & 147 & 117 & 444 \\
\hline
\end{tabular}


En la tabla $V$ se concreta la relación entre el Resultado del lanzamiento y el Tipo de golpeo $\left(X^{2}=43,147 ; p<0,05\right)$ con un nivel de asociación moderado $(\varphi c=0,312)$.

Tabla V. Relación entre las variables Resultado del lanzamiento y la Tipo de golpeo.

\begin{tabular}{|c|c|c|c|c|c|c|}
\hline \multirow{2}{*}{ Tipo de golpeo } & & \multicolumn{5}{|c|}{ Resultado del lanzamiento } \\
\hline & & Gol & A portería & Fuera & Otros & Total \\
\hline \multirow[t]{3}{*}{ Pie interior } & $\mathrm{n}$ & 27 & 27 & 24 & 26 & 104 \\
\hline & FE & 14,8 & 27,4 & 34,4 & 27,4 & 104,0 \\
\hline & RTC & $3,9^{*}$ & $-0,1$ & $-2,5^{\star}$ & $-0,4$ & \\
\hline \multirow{3}{*}{ Pie empeine } & $\mathrm{n}$ & 19 & 60 & 77 & 67 & 223 \\
\hline & FE & 31,6 & 58,8 & 73,8 & 58,8 & 223,0 \\
\hline & RTC & $-3,4^{\star}$ & 0,3 & 0,6 & 1,8 & \\
\hline \multirow[t]{3}{*}{ Pie puntera } & $\mathrm{n}$ & 8 & 20 & 27 & 18 & 73 \\
\hline & FE & 10,4 & 19,2 & 24,2 & 19,2 & 73,0 \\
\hline & RTC & $-0,9$ & 0,2 & 0,8 & $-0,4$ & \\
\hline \multirow[t]{3}{*}{ Pie exterior } & $\mathrm{n}$ & 2 & 3 & 4 & 2 & 12 \\
\hline & FE & 1,7 & 3.2 & 4,0 & 3,2 & 12,0 \\
\hline & RTC & 0,2 & $-0,1$ & 0,0 & $-0,1$ & \\
\hline \multirow{3}{*}{ Pie tacón } & $\mathrm{n}$ & 3 & 1 & 0 & 0 & 4 \\
\hline & FE & 0,6 & 1,1 & 1,3 & 1,1 & 4,0 \\
\hline & RTC & $3,5^{\star}$ & $-0,1$ & $-1,4$ & $-1,2$ & \\
\hline \multirow[t]{3}{*}{ Cabeza frontal } & $\mathrm{n}$ & 3 & 3 & 8 & 2 & 16 \\
\hline & FE & 2,3 & 4,2 & 5,3 & 4,2 & 16,00 \\
\hline & RTC & 0,5 & $-0,7$ & 1,5 & $-1,3$ & \\
\hline \multirow[t]{3}{*}{ Cabeza lateral } & $n$ & 0 & 0 & 1 & 1 & 2 \\
\hline & FE & 0,3 & 0,5 & 0,7 & 0,5 & 2,0 \\
\hline & RTC & $-0,6$ & $-0,8$ & 0,5 & 0,8 & \\
\hline Total & $n$ & 63 & 117 & 147 & 117 & 444 \\
\hline
\end{tabular}

Los resultados muestran que los lanzamientos que se realizan con el Pie interior tienen una probabilidad mayor de lo esperado de terminar en $\mathrm{Gol}$ ( $R T C=3,9 ; n=27 ; F E=14,8)$ junto con una menor probabilidad de lo esperado de que vayan Fuera de la portería ( $R T C=-2,5 ; n=24 ; F E=34,4)$, mientras que los lanzamientos que se realizan con el Pie Empeine muestran una menor probabilidad de lo esperado de acabar en $G o l(R T C=-3,4 ; n=19 ; F E=31,6)$. Además, existe una mayor probabilidad de lo esperado de que los lanzamientos que se realizan con el Tacón del pie acaben en $G o l(R T C=3,5 ; n=3 ; F E=0,6)$, pero este resultado no se puede considerar relevante ya que tan solo se registraron 4 lanzamientos de este tipo.

A continuación, en la tabla VI se detalla las características de la vinculación entre las variables Resultado del lanzamiento y Altura del lanzamiento $\left(X^{2}=61,181 ; p<0,05\right)$ que tiene un nivel de asociación moderado $(\varphi C=0,374)$. 
Tabla VI. Relación entre las variables Resultado del lanzamiento y la Altura del lanzamiento.

\begin{tabular}{|c|c|c|c|c|c|c|}
\hline \multirow[b]{2}{*}{$\begin{array}{c}\text { Altura del } \\
\text { lanzamiento }\end{array}$} & & \multicolumn{5}{|c|}{ Resultado del lanzamiento } \\
\hline & & Gol & A portería & Fuera & Otros & Total \\
\hline Bajo & $\begin{array}{l}\mathrm{n} \\
\mathrm{FE} \\
\mathrm{RTC}\end{array}$ & $\begin{array}{r}35 \\
29,7 \\
1.5\end{array}$ & $\begin{array}{r}56 \\
55,1 \\
0.2\end{array}$ & $\begin{array}{r}44 \\
69,2 \\
-5,1^{*}\end{array}$ & $\begin{array}{r}74 \\
55,1 \\
4.1^{*}\end{array}$ & $\begin{array}{r}209 \\
209,0\end{array}$ \\
\hline Media altura & $\begin{array}{l}\mathrm{n} \\
\mathrm{FE} \\
\mathrm{RTC}\end{array}$ & $\begin{array}{r}16 \\
13,9 \\
0,7\end{array}$ & $\begin{array}{r}26 \\
25,8 \\
0,0\end{array}$ & $\begin{array}{r}25 \\
32,4 \\
-1,8\end{array}$ & $\begin{array}{r}31 \\
25,8 \\
1,3\end{array}$ & $\begin{array}{r}98 \\
98,00\end{array}$ \\
\hline Alto & $\begin{array}{l}\mathrm{n} \\
\mathrm{FE} \\
\mathrm{RTC}\end{array}$ & $\begin{array}{r}12 \\
19,4 \\
-\mathbf{2}, \mathbf{2}^{*}\end{array}$ & $\begin{array}{r}35 \\
36,1 \\
-0,3\end{array}$ & $\begin{array}{r}78 \\
45,4 \\
7,1^{*}\end{array}$ & $\begin{array}{r}12 \\
36,1 \\
-5,6^{*}\end{array}$ & $\begin{array}{r}137 \\
137,0\end{array}$ \\
\hline Total & $\mathrm{n}$ & 63 & 117 & 147 & 117 & 444 \\
\hline
\end{tabular}

Como se puede apreciar, refleja una menor probabilidad de lo esperado de que los lanzamientos Bajos se dirijan Fuera de la portería (RTC=-5,1; $n=44$; $F E=69,2$ ) con una mayor probabilidad de lo esperado de que se produzcan Otro tipo de resultados ( $R T C=4,1 ; n=74 ; F E=55,1)$. Con los lanzamientos Altos existe una menor probabilidad de lo esperado de que acaben en $\mathrm{Gol}(R T C=-2,2 ; n=12$; $F E=19,4)$, de que se produzca Otro tipo de resultado ( $R T C=-5,6 ; n=12 ; F E=36,1)$ junto a esto hay una probabilidad mayor de lo esperado de que el balón termine Fuera $(R T C=7,1 ; n=78 ; F E=45,4)$.

La información en torno a la asociación moderada $(\varphi c=0,430)$ entre el Resultado de lanzamiento y la Oposición en el lanzamiento ( $\left.X^{2}=82,090 ; p<0,05\right)$, se muestra en la tabla VII.

Tabla VII. Relación entre las variables Resultado del lanzamiento y la Oposición en el lanzamiento.

\begin{tabular}{|c|c|c|c|c|c|c|}
\hline \multirow{2}{*}{ Oposición } & & \multicolumn{5}{|c|}{ Resultado del lanzamiento } \\
\hline & & Gol & A portería & Fuera & Otros & Total \\
\hline \multirow[t]{3}{*}{ Sin oposición } & $\mathrm{n}$ & 9 & 0 & 2 & 0 & 11 \\
\hline & FE & 1,6 & 2,9 & 3,6 & 2,9 & 11.0 \\
\hline & RTC & $6,5^{\star}$ & $-2,0^{\star}$ & $-1,1$ & $-2,0^{*}$ & \\
\hline \multirow[t]{3}{*}{ Portero } & $\mathrm{n}$ & 23 & 31 & 29 & 4 & 87 \\
\hline & FE & 12,3 & 22,9 & 28,8 & 22,9 & 87,0 \\
\hline & RTC & $3,7^{\star}$ & $2,2^{*}$ & 0,0 & $-5,1^{*}$ & \\
\hline \multirow{3}{*}{ Oposición lejana } & $\mathrm{n}$ & 13 & 36 & 46 & 48 & 143 \\
\hline & FE & 20,3 & 37,7 & 47,3 & 37,7 & 143,0 \\
\hline & RTC & $-2,1^{*}$ & -04 & $-0,3$ & $2,4^{\star}$ & \\
\hline \multirow[t]{3}{*}{ Oposición cercana } & $\mathrm{n}$ & 18 & 50 & 70 & 65 & 203 \\
\hline & FE & 28,8 & 53,5 & 67,2 & 53,5 & 203,0 \\
\hline & RTC & $-2,9^{\star}$ & $-0,8$ & 0,6 & $2,5^{\star}$ & \\
\hline Total & $\mathrm{n}$ & 63 & 117 & 147 & 117 & 444 \\
\hline
\end{tabular}

En los encuentros analizados, cuando se lanza Sin oposición hay una mayor probabilidad de lo esperado de conseguir $G o l(R T C=6,5 ; n=9 ; F E=1,6)$, al igual que, en el uno contra uno, hacia el Portero ( $R T C=3,7 ; n=23 ; F E=12,3)$, que además tiene una probabilidad mayor de lo esperado de que el balón vaya a portería ( $R T C=2,2 ; n=31 ; F E=22,9$ ) y una menor probabilidad de lo esperado de que ocurra Otro tipo de resultado ( $R T C=-5,1 ; n=4 ; F E=22,9$ ). Cuando se producen lanzamientos con Oposición, tanto lejana como cercana, hay una menor probabilidad de obtener Gol (RTC=-2,1; $n=13 ; F E=20,3$ y $R T C=-2,9$; 
$n=18 ; F E=28,8)$, pero con una mayor probabilidad de lo esperado de que ocurra Otro tipo de resultados ( $R T C=2,4 ; n=48 ; F E=37,7$ y $R T C=2,5 ; n=65 ; F E=53,5)$.

Por último, como se puede apreciar en la tabla VIII, la relación entre el Resultado del lanzamiento y la Situación de juego $\left(\mathrm{X}^{2}=24,662 ; p<0,05\right)$ tiene un nivel de asociación bajo $(\varphi c=0,236)$.

Tabla VIII. Relación entre las variables Resultado del lanzamiento y la Situación de juego.

\begin{tabular}{llrrrrr}
\hline & & \multicolumn{5}{c}{ Resultado del lanzamiento } \\
\cline { 3 - 7 } Situación de juego & & Gol & A portería & Fuera & Otros & \multicolumn{1}{c}{ Total } \\
\hline En juego & $\mathrm{n}$ & 59 & 111 & 141 & 97 & 408 \\
& $\mathrm{FE}$ & 57,9 & 107,5 & 135,1 & 107,5 & 408,0 \\
\multirow{4}{*}{ Penalti } & $\mathrm{RTC}$ & 0,6 & 1,4 & $\mathbf{2 , 2 ^ { * }}$ & $\mathbf{- 4 , \mathbf { 1 } ^ { * }}$ & \\
\cline { 2 - 7 } & $\mathrm{n}$ & 1 & 0 & 0 & 0 & 1 \\
& $\mathrm{FE}$ & 0,1 & 0,3 & 0,3 & 0,3 & 1,0 \\
Falta & $\mathrm{RTC}$ & $\mathbf{2 , 5 ^ { * }}$ & $-0,6$ & $-0,7$ & $-0,6$ & \\
\cline { 2 - 8 } & $\mathrm{n}$ & 3 & 6 & 6 & 20 & 35 \\
& $\mathrm{FE}$ & 5,0 & 9,2 & 11,6 & 9,2 & 35,0 \\
\hline Total & $\mathrm{RTC}$ & $-1,0$ & $-1,3$ & $\mathbf{- 2 , \mathbf { 1 } ^ { * }}$ & $\mathbf{4 , 3 ^ { * }}$ & \\
\hline & $\mathrm{n}$ & 63 & 117 & 147 & 117 & 444 \\
\hline
\end{tabular}

Los resultados muestran que cuando los lanzamientos se producen estando el balón En juego hay una mayor probabilidad de lo esperado de que se vaya Fuera $(R T C=2,2 ; n=141 ; F E=135,1)$ con una menor probabilidad de lo esperado de que se consiga Otro tipo de resultado ( $R T C=-4,1 ; n=97 ; F E=107,5)$. Sin embargo, en los lanzamientos de Penalti hay una mayor probabilidad de lo esperado de conseguir $\mathrm{Gol}(R T C=2,5 ; n=1 ; F E=0,1)$, sin que este resultado sea muy relevante porque sólo se produce un lanzamiento entre todos los partidos registrados. Por último, en el caso de los lanzamientos de Falta, hay una menor probabilidad de lo esperado de que el balón vaya Fuera $(R T C=-2,1 ; n=6$; $F E=11,6)$, junto con una mayor probabilidad de lo esperado de que se consiga Otro tipo de resultado $(R T C=4,3 ; n=20 ; F E=9,2)$,

\section{DISCUSIÓN}

Desde el punto de vista descriptivo, dos de los tres equipos que más lanzamientos realizaron por partido, son aquellos que más lejos llegaron en la competición: Rusia (primera), Ucrania (segunda), e Irán (tercera). Estos resultados obtenidos ponen de manifiesto que los jugadores con parálisis cerebral se comportan de una manera similar a los jugadores de fútbol sin discapacidad: los equipos que más lanzan a portería son los equipos que mejores resultados tienen en competiciones y torneos (Casáis et al., 2011; Szwarc, 2004).

Otro aspecto relevante es que los jugadores con mejor capacidad funcional (CP8) realizaron casi el mismo número de lanzamientos que los de nivel CP7, a pesar de que sólo hay un jugador clasificado como CP8 por equipo en el campo, mientras que de clasificados como CP7 puede haber hasta cuatro. Esto es similar a lo que ocurre en otros deportes de personas con discapacidad, como es el caso del Baloncesto en silla de ruedas (Molik et al., 2009), y que confirma que en el deporte adaptado la eficiencia en el juego de equipo está muy condicionada por la clasificación funcional del deportista, justificando así la 
existencia de los sistemas de clasificación funcional para asegurar la equidad de la competición. Sin embargo, destaca el hecho de que no hay una probabilidad mayor de lo esperado de que los jugadores con mayor capacidad funcional (CP8) marquen gol, a pesar de que, como se ha comentado anteriormente, lanzan más veces. Esto implica que, en el Fa7PC, el éxito no está condicionado por las acciones individuales de los jugadores con más capacidad funcional, por lo que los entrenadores han de hacer un esfuerzo por mejorar aspectos tácticos, como en el resto de deportes de equipo, que faciliten la obtención del gol.

Con respecto al momento de juego, los resultados ponen de manifiesto que se realizan más lanzamientos al final del partido. Esto podría ser debido al cansancio, ya que los equipos están mucho más desordenados y el juego se hace más rápido, con transiciones más frecuentes de un lugar a otro del campo (Tsitskaris et al., 2002). En función del rol del jugador, son los Delanteros los que muestran un mayor porcentaje de lanzamientos por partido. Los equipos suelen jugar con dos defensas, tres centrocampistas y un delantero (como mucho dos). En Goalball, deporte colectivo específico de personas con discapacidad visual, también se aprecia diferencias en el porcentaje de lanzamientos por partido en función de la posición y el rol del jugador en el terreno de juego (Morato et al., 2017).

Por otro lado, los resultados muestran que existen variables zonales de juego que pueden influir en el rendimiento en los lanzamientos de Fa7PC. Cuando los lanzamientos se producen desde la Zona 2, Zona 3 y Zona 6 del campo, existe una mayor probabilidad de lo esperado de que el resultado sea Gol. Esto podría ser debido a que son las zonas centrales del campo, mientras que, si se lanza desde la Zona 1, contigua a la línea de fondo y muy esquinada, la probabilidad de que el lanzamiento vaya Fuera, es mayor de lo esperado. Además, si el lanzamiento se produce desde la Zona 9 o la Zona 14 existe una mayor probabilidad de lo esperado de que se produzca Otro tipo de resultado. Estas son zonas alejadas de la portería, donde siempre suele haber algún jugador (defensa o delantero) en la trayectoria del lanzamiento. Además, desde la Zona 14 hay menor probabilidad de lo esperado de que el lanzamiento sea Gol o Fuera, debido a que esta también es una zona muy centrada en el campo. Los resultados son similares a algunos estudios previos en otros deportes, como es el caso del Floorball (Prieto et al., 2013) o del Baloncesto (Ibáñez et al., 2009) que muestran que los lanzamientos que se producen en las zonas más cercanas al punto de tanteo (porterías o canastas) son los que tienen más probabilidad de éxito.

Las características del contexto deportivo del Fa7PC condicionan la eficacia de la acción final, al igual que en otros deportes cooperativos, como es el caso de Hockey hierba (Piñero, 2008) o del Floorball (Prieto et al., 2013), donde el lanzamiento depende de acciones técnicas previas como el pase. Si el lanzamiento se ejecuta con el Interior del pie, hay una mayor probabilidad de conseguir Gol. Este tipo de lanzamiento se utiliza en zonas cercanas a la portería para ajustar más la dirección del balón. Sin embargo, cuando se lanza con el Empeine, existe una menor probabilidad de lo esperado de conseguir Gol, ya que este tipo de lanzamiento se suele utilizar en disparos lejanos y potentes, pero menos controlados. Esto contrasta con el hecho de que los jugadores 
emplean más el empeine en sus golpeos, a pesar de que este tipo de lanzamiento no muestra una probabilidad mayor de lo esperado de obtener éxito.

También se observa que hay menor probabilidad de lo esperado de que los lanzamientos Bajos vayan Fuera, junto con una mayor probabilidad de lo esperado de que obtengan Otro tipo de resultado; puede ser porque a esa altura se produzcan más rechaces. Por otro lado. los lanzamientos Altos tienen menor probabilidad de lo esperado de acabar en Gol o con Otro tipo de resultado, debido a la mayor probabilidad de lo esperado de que vayan Fuera. Esto es similar a lo que sucede en otros deportes de personas con discapacidad, como el Goalball, en el que los resultados muestran que los lanzamientos en función de la altura a la que se realicen tienen mayor o menor eficacia (Morato et al., 2017).

Además, en Fa7PC cuando un jugador lanza Sin oposición o a solas con el Portero hay mayor probabilidad de lo esperado de que el resultado sea Gol (o que vaya $A$ portería, en el caso de la categoría Portero). Es algo coherente puesto que los tiros se producen sin presión defensiva, y que permite comprobar que el estudio se corresponde con la realidad del juego. Sin embargo, cuando los lanzamientos se producen con Oposición lejana o cercana, hay menor probabilidad de lo esperado de que acaben en Gol pero mayor probabilidad de lo esperado de que se produzca Otro tipo de resultado; En Fa7PC es frecuente que haya jugadores en la trayectoria del balón a la portería, y en concreto, en el momento del lanzamiento hay jugadores en la defensa con intención de interceptar el lanzamiento, al igual que ocurre en Baloncesto (lbáñez et al., 2009), donde la eficacia aumenta considerablemente cuando la presión defensiva es menor o nula.

Por último, cuando el lanzamiento se produce con el balón En juego, hay una mayor probabilidad de lo esperado de que vaya Fuera, al contrario de lo que ocurre cuando se realiza un lanzamiento de Falta, donde la probabilidad de que el balón vaya Fuera es menor de lo esperado y que se produzca Otro tipo de resultado tiene una probabilidad mayor de lo esperado; esto parece ser consecuencia de que la mayoría de los lanzamientos de falta impactan en los jugadores de la barrera.

\section{CONCLUSIÓN}

En la modalidad deportiva del Fa7PC de élite se producen un número elevado de lanzamientos, sobre todo en los últimos minutos, siendo los equipos mejor clasificados los que más lanzamientos realizan por partido. Las zonas centrales del campo son las más empleadas para realizar los disparos a puerta y se observa un equilibrio entre los que finalizan dentro de los tres palos y los que van fuera. Igualmente, el mayor número de lanzamientos a portería los realizan los jugadores delanteros con más capacidad funcional (CP8), a pesar de que no hay una mayor probabilidad de éxito con respecto al resto de jugadores del equipo.

El pie derecho y el empeine son los más utilizados en los lanzamientos a portería, que con más frecuencia tienen trayectorias bajas y se producen con 
oposición cercana. Sin embargo, solo los lanzamientos realizados con el interior del pie tienen una probabilidad mayor de lo esperado de acabar en gol.

Por tanto, se puede concluir que el lanzamiento con la mayor probabilidad de éxito sería aquel que se realiza desde las Zona 2, Zona 3 o Zona 6 del campo, con el interior del pie, una altura baja y sin oposición defensiva o sólo frente al portero.

\section{LIMITACIONES}

La principal limitación del presente trabajo consiste en la escasa documentación científica existente en contraste con el gran número de artículos presente en la literatura en torno al fútbol convencional.

\section{REFERENCIA BIBLIOGRÁFICAS}

Andrade, M., Fleury, A., \& Silva, A. (2005). Isokinetic muscular strength of paralympic athletes with cerebral palsy (CP) fron the Brazilian soccer team. Revista Brasileira de Medicina do Esporte, 11(5), 281-285. http://dx.doi.org/10.1590/S1517-86922005000500007

Anguera, M.T. (1991). Metodología observacional en la investigación psicológica. Barcelona: PPU.

Anguera, M.T., \& Hernández-Mendo, A.H. (2013). Metodología observacional en el ámbito del deporte. E-balonmano.com: Revista de Ciencias del Deporte, 9(3), 135-160.

Armatas, V., Yiannakos, A., Zaggelidis, G., Skoufas, D., Papadopoulou, S., \& Fragkos, N., (2009). Differences in offensive actions between top and last teams in Greek first soccer división. A retrospective study 1998-2008. Journal of Physical Education and Sport, 23(2), 1-5.

Boyd, R.N., \& Graham, H.K. (1999). Objetive measurement of clinical findings in the use of botulinum toxin type. A for the managment of children with cerebral palsy. European Journal of Neurology, 6(4), 23-35.

Bruyère, S., VanLooy, S., \& Peterson, D. (2005). The International Classification of Functioning, Disability and Health (ICF): Contemporary literatura overview. Rehabilitation Psychology, 50, 1-21.

Casáis, L., Lago, C., Lago, J., Iglesias, S., \& Gómez, M. (2011). Indicadores de rendimiento competitivo que diferencia equipos ganadores y perdedores de la Liga Española. Futbolpf: Revista de Preparación Física en el Fútbol, (2), 44-53.

Cohen, J. (1960). A coefficient of agreement for nominal scales. Educational and Psychological $\quad$ Measurement, 20(1), 37-46. https://doi.org/10.1177/001316446002000104

CPISRA - Cerebral Palsy International Sports and Recreation Association. (2014). Football 7-a-side Rules \& Regulations for CPISRA Sanctioned Tournaments and Amendments to the FIFA Laws of the Game. Consultado en http://cpisra.org/main/wp-content/uploads/2014/02/Rules-January-1st2014_2ss.pdf

Crewson, P. (2006). Applied statistics handbook. version 1.2. AcaStat Software. Consultado en http://www.acastat.com/Statbook/contents.htm. 
Drust, B. (2010). Performance analysis research: Meeting the challenge. Journal of Sport Sciences, 28(9), 921-922. https://doi.org/10.1080/02640411003740769

Gamonales, J.M., León, K., Muñoz, J., González-Espinosa, S. \& Ibáñez, S.J. (2018) Validación del IOLF5C para la eficacia del lanzamiento en fútbol para ciegos. Revista Internacional de Medicina y Ciencias de la Actividad Física $y$ el Deporte, 18(70), 361-381. http://dx.doi.org/10.15366/rimcafd2018.70.010

García-Ribés, A. (2004). Aplicación de toxina botulínica tipo A en la parálisis cerebral infantil espástica. Boletín de la Sociedad Vasco-Navarra de Pediatría, 37, 38-43.

Hileno, R., \& Buscá, B. (2012). Herramienta observacional para analizar la cobertura del ataque en voleibol. Revista Internacional de Medicina y Ciencias de la Actividad Física y el Deporte, 12(47), 557-570.

Hughes, M.D., \& Bartlett, R.M. (2008). The use of performance indicators in performance analysis. Journal of Sports Sciences, 20(10), 739-754. https://doi.org/10.1080/026404102320675602

Hughes, M.D., \& Franks, I. (2004). Notational analysis of sport. Systems for better coaching and performance in sport ( $2^{\mathrm{a}}$ ed.). Nueva York: Routledge.

Hughes, M.D., \& Franks, I. (2008). The essentials of Notational Analysis. An introduction. Nueva York: Routledge.

Ibáñez, S.J., García, J., Cañadas, M., Moreno, M.L., Lorenzo, A., \& Gómez, M. A. (2007). Estudio de la eficacia del lanzamiento a canasta en la liga EBA. Revista Portuguesa de Ciencias Do Desporto, 7(1), 78.

Ibáñez, S.J., García, J., Feu, S., Parejo, I., \& Cañada, M. (2009), La eficacia del lanzamiento a canasta en la NBA: análisis multifactorial. Cultura, Ciencia y Deporte, 10, 39-47. http://dx.doi.org/10.12800/ccd.v4i10.132

Ibáñez, S.J., Martínez, B., \& Lozano, A. (2001). Estudio de la tipología de pase y su eficacia durante el juego real en Baloncesto. En Tavares, F., Janeira, M., Graça, A., Pinto, D., y Brandao, E. (Ed.), Tendências actuais investigação em basquetebol (pp. 159-172). Porto: Ediciones FCDEF.

IFCPF - International Federation of Cerebral Palsy Football. (2016). Classification rulebook. Consultado en http://goo.gl/kvL0el

Kloyiam, S., Breen, S., Jakeman, P., Conway, J., \& Hutzler, Y. (2011). Soccerspecific endurance and running economy in soccer players with cerebral palsy. Adapted Physical Activity Quarterly, 28(4), 354-367.

Lago, C. (2005). Ganar o perder en el fútbol de alto nivel: ¿una cuestión de suerte?. Motricidad: Revista de Ciencias de la Actividad Física y del Deporte, (14), 135-150.

Lago, C. (2008). El análisis del rendimiento en los deportes de equipo. Algunas consideraciones metodológicas. Acción Motriz, 1(2), 41-58.

Lago, C., Casáis, L., Domínguez, E., Lago, J., \& Rey, E. (2009). Influencia de las variables contextuales en el rendimiento físico en el fútbol de alto nivel. Motricidad: Revista de Ciencias de la Actividad Física y del Deporte, (23), 107-121.

Manzano, A., Pacheco, J.L., \& Lorenzo, A. (2006). Análisis de la influencia del número de pases, los balones jugados dentro de la zona y la duración como variables de competición en la eficacia de las posiciones en el baloncesto. Estudos, 6, 152-165. 
Mendes, L. \& Tavares, F. (2004). Análise das acçôes defensivas no basketebol. Apresentação de um protocolo de classificação e registro das observações. Estudos, 4, 59- 65.

Molik, B., Kosmol, A., Morgulec-Adamowicz, N., Laskin, J.J., Jezior, T., \& Patrzatek, M. (2009). Game efficiency of elite female wheelchair basketball players during World championships (Gold Cup) 2006. European Journal of Adapted Psysical Activity, 26-38. http://dx.doi.org/10.5507/euj.2009.007

Montero, I.,G., \& León O. (2007). A guide for naming research studies in Phychology. International Journal of Clinical and Health Psychology, 7(3), 847-862.

Morato, M., Da Cunha, O., Gamero, D., Magalhães, T., \& Almeida, J. (2017). Development and evaluation of an observational system for goalball match analysis. Revista Brasileira de Ciências do Esporte, 39(4), 398-407. http://dx.doi.org/10.1016/j.rbce.08.002

O'Donoghue, P. (2010). Research methods for sports performance analysis. London: Routledge.

Pardo, A. (2002). Análisis de datos categóricos. Madrid: Universidad Nacional de Educación a Distancia.

Piñero, R. (2008). Observación y análisis de la acción de gol en hockey hierba. Sevilla: Wanceulen.

Prieto, M., Pérez, J., \& Gómez, M. A. (2013). Indicadores de rendimiento ofensivo en el floorball de alto nivel. Revista Internacional de Ciencias del Deporte, 9(32), 114-125. http://dx.doi.org/10.5232/ricyde2013.03202

Randolph, J. J. (2005). Free-Marginal Multirater Kappa (multirater Kfree): An Alternative to Fleiss' Fixed-Marginal Multirater Kappa. Joensuu Learning and Instruction Symposium 2005, University of the Joensuu, Finland.

Reina, R. (2014). Evidence-based classification in paralympic sport: application to football 7-a-side. European Journal of Human Movement, 32, 161-185.

Roquetti, P., \& Fernandes, J. (2004). Estudio comparativo de la dermatoglifía, somatotipia y del consumo máximo de oxígeno de los atletas de la Selección Brasilera de Football de Campo, Portadores de Parálisis cerebral y de atletas profesionales de Football de Campo, no Portadores de Parálisis Cerebral. Journal Fitness \& Performance, 3(3), 157-164. http://dx.doi.org/10.3900/fpj.3.3.157.s

Salas, J., \& Hernández-Mendo, A. (2016). Análisis de la calidad del dato y generalizabilidad de un sistema de observación del contraataque en el balonmano de élite. E-balonmano.com: Revista de Ciencias del Deporte, 12(1), 31-44.

Szwarc, A. (2004). Effectivenes of Brazilian and German teams and the teams defeated by them during the 17th FIFA World Cup. Kinesiolgy, 36(1), 83-89.

Tsitskaris, G., Theoharopoulos, A., Galanis, D., \& Nikopoulou, M. (2002). Types of shots used at the Greek National Basketball Championship according to the división and position of players. Journal of Human Movement Studies, 42, 43-52.

Tweedy, S., \& Valandewijck, Y. (2011). International Paralympic committee position stand-background and scientific principles of classification in Paralympic sport. British Journal of Sports Medicine, 45, 259-269. http://dx.doi.org/10.1136/bjsm.2009.065060 
Villarejo, D., Ortega, E., Gómez, M.A., \& Palao, J. (2014). Design, validation and reliability of an observational instrument for ball possessions in rugby unión. International Journal of Performance Analysis, 14, 896-908. https://doi.org/10.1080/24748668.2014.11868771

Wheeler, K.W., Askew, C.D., \& Sayers, M.G.L. (2010). Effective attacking strategies in rugby union. European Journal of Sports Science, 10(4), 237242. https://doi.org/10.1080/17461391.2010.482595

Yanci, J., Los Arcos, A., Grande, I., Santalla, A., Figueroa, J., Gil, E., \& Cámara, J. (2014). Capacidad de salto en futbolistas con parálisis cerebral. Revista Internacional de Medicina y Ciencias de la Actividad Física y el Deporte, 14(54), 199-211.

Referencias totales / Total references: 44 (100\%)

Referencias propias de la revista /Journal's own references: $3(6,82 \%)$

Rev.int.med.cienc.act.fís.deporte - vol. 19 - número 74 - ISSN: 1577-0354 